\title{
Fast collisionless reconnection and electron heating in strongly magnetized plasmas
}

\author{
N. F. Loureiro, ${ }^{1}$ A. A. Schekochihin ${ }^{2}$ and A. Zocco ${ }^{3,2}$ \\ ${ }^{1}$ Associação EURATOM/IST, Instituto de Plasmas e Fusão Nuclear - Laboratório Associado, \\ Instituto Superior Técnico, Universidade Técnica de Lisboa, 1049-001 Lisboa, Portugal \\ ${ }^{2}$ Rudolf Peierls Centre for Theoretical Physics, University of Oxford, Oxford OX1 3NP, UK \\ ${ }^{3}$ EURATOM/CCFE Fusion Association, Culham Science Centre, Abingdon OX14 3DB, UK
}

(Dated: May 5, 2018)

\begin{abstract}
Magnetic reconnection in strongly magnetized (low-beta), weakly collisional plasmas is investigated using a novel fluid-kinetic model [Zocco \& Schekochihin, Phys. Plasmas 18, 102309 (2011)] which retains non-isothermal electron kinetics. It is shown that electron heating via Landau damping (linear phase mixing) is the dominant dissipation mechanism. In time, electron heating occurs after the peak of the reconnection rate; in space, it is concentrated along the separatrices of the magnetic island. For sufficiently large systems, the peak reconnection rate is $c E_{\max } \approx 0.2 v_{A} B_{y, 0}$, where $v_{A}$ is the Alfvén speed based on the reconnecting field $B_{y, 0}$. The island saturation width is the same as in MHD models except for small systems, when it becomes comparable to the kinetic scales.

PACS numbers: 52.35.Vd, 96.60.Iv, 52.35.Py, 52.30.Gz
\end{abstract}

Introduction. Magnetic reconnection is a reconfiguration of the magnetic field in a plasma via localized unfreezing of the magnetic flux [1]. It is commonly associated with energy release in astrophysical and laboratory plasmas, but the details of the energy conversion mechanisms and partition between particle species and fields are poorly understood. This Letter focuses on a key aspect of this issue: the conversion of the magnetic energy into electron internal energy, i.e., electron heating.

We consider a fundamental reconnection paradigm: the tearing mode in a periodic box [2]. The tearing instability leads to the opening, growth and saturation of a magnetic island [2-6]. Since the saturated width of the island is in general macroscopic, it cannot depend on the microphysics of the plasma and, in particular, should not depend on its collisionality (as we will show). This implies that the total fraction of the initial magnetic energy converted to other forms of energy from the begining to the end of the evolution of the tearing mode must be the same in collisional and collisionless plasmas. In a periodic (closed) system, energy cannot be lost via bulk plasma outflows. Therefore, the magnetic energy difference between the initial and final states must be accounted for by conversion into the thermal energy of the particles. In collisional plasmas, this is achieved by Ohmic and viscous heating [7]. However, many natural systems where reconnection occurs are only weakly collisional; the only available heating channels then are Landau damping and electron viscosity, both of which ultimately rely on the electron collision frequency being finite, though possibly arbitrarily small. In this Letter, we show that electron heating via linear phase mixing associated with Landau damping is the main energy conversion channel in weakly collisional reconnection in strongly magnetized (low-beta) plasmas.

Equations. We use a fluid-kinetic approximation applicable to low-beta plasmas ("KREHM" [8]). Its main distinctive feature, for our purposes, is the coupling of Ohm's law to the electron (drift) kinetic equation via the electron temperature fluctuations. The kinetic equation allows for both collisions and Landau resonance, thus enabling "collisionless" electron heating.

We work in the low- $\beta$ regime, where $\beta$, the ratio of the plasma to the magnetic pressure, is ordered similar to the electron-ion mass ratio $m_{e} / m_{i}$. Let us define the perturbed electron distribution function to lowest order in $\sqrt{m_{e} / m_{i}} \sim \sqrt{\beta}$, and in the gyrokinetic expansion $[9$ 12] as $\delta f_{e}=g_{e}+\left(\delta n_{e} / n_{0 e}+2 v_{\|} u_{\| e} / v_{\text {the }}^{2}\right) F_{0 e}$, where $F_{0 e}$ is the equilibrium Maxwellian, $v_{\text {the }}=\sqrt{2 T_{0 e} / m_{e}}$ is the electron thermal speed (with $T_{0 e}$ the mean electron temperature), $v_{\|}$is the parallel velocity coordinate, $\delta n_{e} / n_{0 e}$ is the electron density perturbation (the zeroth moment of $\delta f_{e}$ ) normalized to its background value $n_{0 e}$, and $u_{\| e}=\left(e / \mathrm{cm}_{e}\right) d_{e}^{2} \nabla_{\perp}^{2} A_{\|}$is the parallel electron flow (the first moment of $\delta f_{e} ; A_{\|}$is the parallel component of the vector potential and $d_{e}=c / \omega_{p e}$ is the electron skin depth). All moments of $\delta f_{e}$ higher than $\delta n_{e}$ and $u_{\| e}$ are contained in $g_{e}$, e.g., the electron temperature perturbation is $\delta T_{\| e} / T_{0 e}=\left(1 / n_{0 e}\right) \int d^{3} \mathbf{v}\left(2 v_{\|}^{2} / v_{\text {the }}^{2}\right) g_{e}$. The dynamics of the plasma is described by the evolution equations for $\delta n_{e} / n_{0 e}, A_{\|}$and $g_{e}$, which, for the 2D case considered here, read [8]:

$$
\begin{aligned}
& \frac{1}{n_{0}} \frac{d \delta n_{e}}{d t}=\frac{1}{B_{z}}\left\{A_{\|}, \frac{e}{c m_{e}} d_{e}^{2} \nabla_{\perp}^{2} A_{\|}\right\}, \\
& \frac{d}{d t}\left(A_{\|}-d_{e}^{2} \nabla_{\perp}^{2} A_{\|}\right)=-\frac{c T_{0 e}}{e B_{z}}\left\{A_{\|}, \frac{\delta n_{e}}{n_{0 e}}+\frac{\delta T_{\| e}}{T_{0 e}}\right\}, \\
& \frac{d g_{e}}{d t}-\frac{v_{\|}}{B_{z}}\left\{A_{\|}, g_{e}-\frac{\delta T_{\| e}}{T_{0 e}} F_{0 e}\right\}=C\left[g_{e}\right] \\
&-\left(1-\frac{2 v_{\|}^{2}}{v_{\text {the }}^{2}}\right) \frac{F_{0 e}}{B_{z}}\left\{A_{\|}, \frac{e}{c m_{e}} d_{e}^{2} \nabla_{\perp}^{2} A_{\|}\right\},
\end{aligned}
$$

where $C\left[g_{e}\right]$ is the collision operator $[13],\{\ldots, \ldots\}$ is the 
Poisson bracket and $d / d t=\partial / \partial t+c / B_{z}\{\varphi, \ldots\}$, with $B_{z}$ the out-of-plane magnetic guide-field and $\varphi$ the electrostatic potential. The latter is obtained via the gyrokinetic Poisson's law [10], $\delta n_{e} / n_{0 e}=1 / \tau\left(\hat{\Gamma}_{0}-1\right) e \varphi / T_{0 e}$, where $\tau=T_{0 i} / T_{0 e}$ and $\hat{\Gamma}_{0}$ is the real-space operator that is the inverse Fourier transform of $\Gamma_{0}(\alpha)=I_{0}(\alpha) e^{-\alpha}$, with $I_{0}$ the modified Bessel function and $\alpha=k_{\perp}^{2} \rho_{i}^{2} / 2$ ( $\rho_{i}=v_{\text {th } i} / \Omega_{i}$ is the ion Larmor radius).

Eq. (3) shows that the popular isothermal closure [14], $g_{e}=0$, is not a solution of that equation unless $\left\{A_{\|}, \nabla_{\perp}^{2} A_{\|}\right\}=0$, a condition that cannot describe a reconnecting system [though it does describe the (macroscopic) island saturation [4, 5], as we will find]. This means that at least the possibility of electron heating in weakly collisional reconnection cannot be ignored.

Numerical Details. To simplify the solution of Eq. (3), note that it does not contain an explicit dependence on the perpendicular velocity coordinate, $v_{\perp}$. If we ignore any such a dependence that is introduced by the collision operator, $v_{\perp}$ can be integrated out, so $g_{e}=g_{e}\left(x, y, v_{\|}, t\right)$. Next, we introduce the Hermite expansion $g_{e}\left(x, y, t, v_{\|}\right)=$ $\sum_{m=2}^{\infty} H_{m}\left(v_{\|} / v_{\text {the }}\right) g_{m}(x, y, t) F_{0 e}\left(v_{\|}\right) / \sqrt{2^{m} m !}$

$\left(g_{0}=g_{1}=0\right.$ because $\delta n_{e}$ and $u_{\| e}$ have been explicitly separated in the decomposition of $\delta f_{e}$ adopted above). Eq. (3) then unfolds into a series of coupled, fluid-like equations for each of the coefficients $g_{m}$ :

$$
\begin{gathered}
\frac{d g_{m}}{d t}=\frac{v_{\text {the }}}{B_{z}}\left(\sqrt{\frac{m+1}{2}}\left\{A_{\|}, g_{m+1}\right\}+\sqrt{\frac{m}{2}}\left\{A_{\|}, g_{m-1}\right\}\right) \\
+\frac{\sqrt{2}}{B_{z}} \delta_{m, 2}\left\{A_{\|}, \frac{e}{c m_{e}} d_{e}^{2} \nabla_{\perp}^{2} A_{\|}\right\}-\nu_{\text {coll }} m^{4} g_{m},
\end{gathered}
$$

where we have adopted a model (hyper) collision operator with $\nu_{\text {coll }}=1 /\left(\Delta t M^{4}\right)$, where $M$ is the index of the highest Hermite polynomial kept in a simulation, and $\Delta t$ the timestep. Thus, in our simulations, $M$ is a proxy for the collision frequency, with higher values of $M$ corresponding to less collisional systems (at the large values of $M$ reported here, $\Delta t \sim M^{-1 / 2}$ so $\nu_{\text {coll }} \sim M^{-7 / 2}$ ). Note that the more familiar (and more physical) in such 1D problems Lénard-Bernstein collision operator would instead be $m \nu_{e i} g_{m}$ [8] ( $\nu_{e i}$ is the electron-ion collision frequency); because of the linear dependence on $m$ of this operator an unfeasibly large number of Hermite polynomials would need to be kept in order to resolve the velocity-space cutoff as $\nu_{e i} \rightarrow 0$. This is why we use hyper-collisions instead.

Eqs. $(1,2,4)$ are solved numerically using a pseudospectral code [15]. The spatial grid size is $384^{2}$. The resolution in velocity space is set by $M$ and ranges from 30 to 500. Hyper-diffusive terms of the form $\nu_{H} \nabla_{\perp}^{6}$, where $\nu_{H}=0.25 / \Delta t(\Delta x / \pi)^{6}$, with $\Delta x$ the grid spacing, are added to the RHS of all equations (including Eq. (4)). These are required to prevent the unbounded thinning of the current layer in the nonlinear regime [16-18],

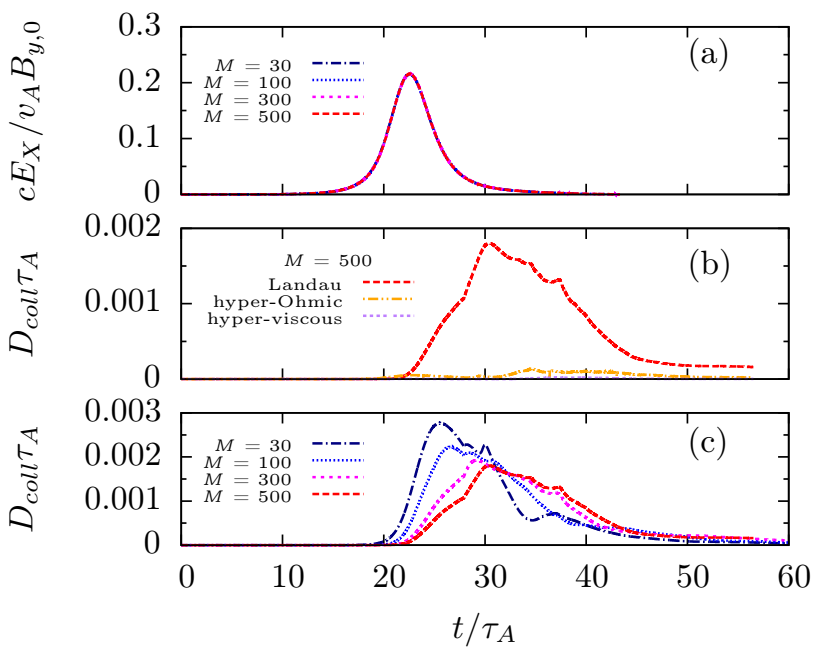

FIG. 1. Time traces of: (a) the reconnection rate for different values of collisionality (represented by $M$; larger $M$ means less collisions); (b) the rates of dissipation via Landau damping and hyper-dissipation, for the least collisional case $(M=500)$; (c) the rate of dissipation via Landau damping for different values of $M$. All runs had $\Delta^{\prime} a=20$.

but will not, as we will discover, dissipate much energy. Physically, they stand in for collisional and collisionless electron finite Larmor radius effects (which are formally small in the KREHM approximation).

The hyper-diffusive and hyper-collisional terms are the only dissipative terms we employ. We shall distinguish their effects: "hyper-viscous" and "hyper-Ohmic" refer, respectively, to the dissipation terms in Eq. (1) and in Eq. (2); "Landau" refers to the dissipation arising via both hyper-collisions and hyper-dissipation in Eq. (4). The rationale for the latter is that the only way that energy can arrive at any $g_{m}$ with $m>2$ is via phase mixing. Once there, how exactly it dissipates is not important: what we wish to investigate is the relative importance of dissipation via phase mixing (i.e., Landau-damping) vs. (hyper-) viscosity or resistivity.

The equilibrium in-plane magnetic field is $B_{y, e q}=$ $-d A_{\| e q} / d x$, with $A_{\| e q}=A_{\| 0} / \cosh ^{2}(x / a)$, where $a$ is the (normalizing) equilibrium scale length. The (normalizing) Alfvén time is defined as $\tau_{A}=a / v_{A}$ with $v_{A}$ the Alfvén speed based on $B_{y, 0}=\max \left|B_{y, e q}\right|=1$. The simulations are performed in a doubly periodic box of dimensions $L_{x} \times L_{y}$, with $L_{x} / a=2 \pi$ and $L_{y}$ such that $\hat{k}_{y}=2 \pi a / L_{y}$ yields the desired value of the tearing instability parameter $\Delta^{\prime} a=2\left(5-\hat{k}_{y}^{2}\right)\left(3+\hat{k}_{y}^{2}\right) /\left(\hat{k}_{y}^{2} \sqrt{4+\hat{k}_{y}^{2}}\right)[6]$. For simplicity and numerical convenience, we set $\rho_{i}=$ $\rho_{s}=d_{e}=0.25 a$, where $\rho_{s}=\rho_{i} / \sqrt{2 \tau}$. Thus, this study does not address any effects associated with the scale separation between ions and electrons - a reasonable first step for low- $\beta$ plasmas.

Reconnection Rate. Before we discuss the electron heating, let us first report some of the more standard 

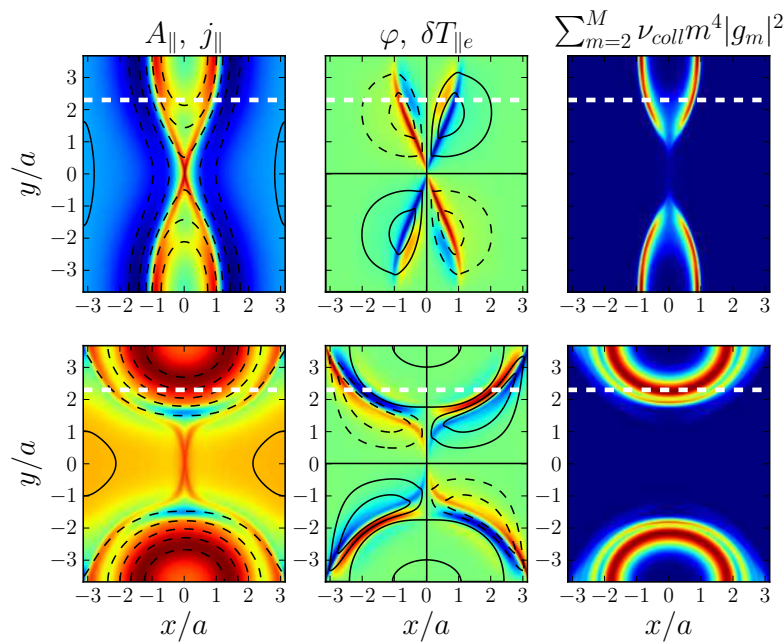

FIG. 2. System configuration for our least collisional simulation $(M=500)$ at the time of maximum reconnection rate (top row) and of maximum dissipation rate (bottom row) [see Figs. 1(a) and (b), respectively]. From left to right, the plots show: $A_{\|}$(lines: full/dashed are positive/negative contours) and $j_{\|}=-c / 4 \pi \nabla_{\perp}^{2} A_{\|}$[colors, ranging from blue (negative) to red (positive) $] ; \varphi$ (lines) and $\delta T_{\| e}$ (colors); the total dissipation via $g_{m}$ 's (i.e., via Landau damping). The horizontal dashed line marks the location of the domain cut where the distribution function of Fig. 4 (bottom row) is plotted.

features of our simulations. The system evolves in time from the linear to the nonlinear stage; saturation occurs when all the initially available flux has been reconnected. The time-traces of the reconnection rate, defined as the value of the parallel electric field at $(x, y)=(0,0)$ (the $X$-point), $E_{X}$, are plotted in Fig. 1(a). As shown, the reconnection rate is entirely independent of collisionality (parameterized by $M$ ), consistent with the fact that our simulations are in the weakly-collisional regime, where the frozen-flux constraint is broken by electron inertia, not the collisions. The maximum value is $c E_{X}^{\max } \approx$ $0.22 v_{A} B_{y, 0}$, similar to the fast reconnection rates obtained in the opposite limit of weak guide-field [19], and in qualitative agreement with [20,21]. Regarding the dependence of $E_{X}^{\max }$ with system size (not shown), we found that $E_{X}^{\max }$ increases with $\Delta^{\prime}$, asymptoting to $c E_{X}^{\max } \sim 0.2 v_{A} B_{y, 0}$ for $\Delta^{\prime} a \gtrsim 10$.

Fig. 2 depicts the system configuration for our least collisional simulation $(M=500)$ at the time of maximum reconnection rate $\left[t / \tau_{A}=22.7\right.$; see Fig. 1 (a) $]$ (top row) and at the time of maximum dissipation rate $\left[t / \tau_{A}=\right.$ 29.4; see Fig. 1(b)] (bottom row). A typical $X$-point geometry is seen, accompanied by a quadrupole structure exhibited by $\delta T_{\| e}$ (and by $\delta n_{e}$, not shown) [22] [23].

Saturation. Fig. 3 shows the saturation amplitudes obtained in our simulations ("KREHM"); overplotted are the $\operatorname{MHD}\left(\rho_{s, i}=d_{e}=0\right)$ results from Ref. [6] ("RMHD"). Also shown is the prediction from MHD theory $[4,5]$ ("POEM"), valid in the small- $\Delta^{\prime} a$ regime.

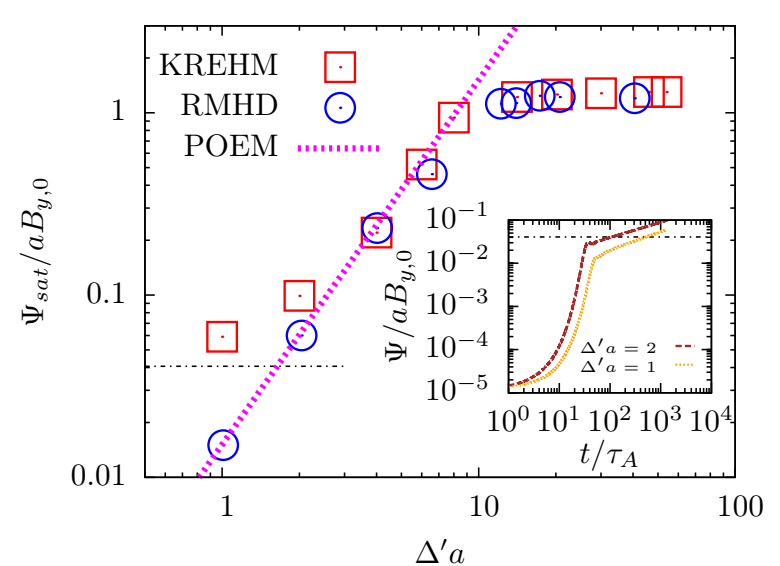

FIG. 3. Saturated flux $\Psi_{\text {sat }}$ vs. $\Delta^{\prime}$. "KREHM" are the data from the kinetic simulations; "RMHD" are MHD results from [6]. The dotted line ("POEM") is the prediction from MHD theory $[4,5]$. Inset: time-traces of flux for $\Delta^{\prime} a=(1,2)$. In both plots, the horizontal line is the saturated flux corresponding to a full island width of $2 d_{e} / a$.

As anticipated, we find that saturation in our weakly collisional, kinetic simulations is well described by the (isothermal) MHD model. At small $\Delta^{\prime} a$, this agreement breaks down because the island saturation amplitude becomes comparable to the kinetic scales $\left(d_{e}, \rho_{s, i}\right)$. Saturation then becomes a slow, diffusive process, as illustrated by the inset plot: the islands slowly expand until their width becomes $\sim d_{e}$. Thus, there is a lower limit to the saturation amplitude, set by $d_{e}$ (cf. [24]); indeed, the frozen-flux condition precludes the definition of magnetic field lines at sub- $d_{e}$ scales.

Electron heating. We have established that the amount of energy converted during the evolution of the system is independent of the collisionality; we have also checked (not shown) that for all but the smallest systems, the energy converted into electron heating is a significant fraction of the initial (magnetic) energy, reaching $\sim 60 \%$ for the largest systems. We now turn to the main focus of this Letter: how is the energy converted. Fig. 1(b) shows the time traces of the dissipation rates for $\Delta^{\prime} a=20$ and $M=500$. We see that dissipation happens almost exclusively via the phase mixing/Landau channel. We reiterate that, a priori, the system is free to choose between different dissipation channels, i.e., the fact that phase mixing is the preferential dissipation mechanism is not hard-wired and is, therefore, a remarkable demonstration of the dominance of Landau damping over other dissipation mechanisms in weakly collisional reconnection [25].

Another noteworthy feature in Fig. 1(b) is the time lag between the peaks of the reconnection and dissipation rates. This implies that magnetic energy is not directly dissipated by the reconnection process itself [26]. Instead, it is first converted to other forms and then dissipated. 
Indeed Landau damping dissipates the electron free energy, $\int d x d y / V \int d v_{\|} T_{0 e} g_{e}^{2} /\left(2 F_{0 e}\right)$. Fig. $1(\mathrm{c})$ shows that the time lag increases weakly with decreasing collisionality (see below).

A related question is where in the domain the heating is occurring. The plots in the right column of Fig. 2 show that there is no significant heating in the current sheet; instead, it happens predominantly along the separatrices of the island. This is consistent with the existence of a time lag between reconnection and dissipation: the magnetic energy that is converted in the current sheet is mostly channeled into ion and electron kinetic energy. Both species flow downstream predominantly along the separatrices. Deceleration of these flows converts kinetic energy into the free energy (or entropy) of the electrons (plotted in the top row of Fig. 4). It is this conversion that constitutes the energetics of Landau damping and results eventually in electron heating.

A detailed understanding of the electron heating process is yielded by the spectral maps [in the twodimensional Fourier-Hermite $\left(k_{\perp}, m\right)$ space] of the electron free energy and its dissipation. These are shown in the two top pannels of Fig. 4 for $M=500$. As time advances, the electron free energy cascades to higher values of $m$, corresponding to the formation of small scales in velocity space, i.e., phase mixing. During this process, energy dissipation occurs via the hyper-diffusive terms, acting at large values of $k_{\perp}$ (see middle pannel, second row). At later times, large enough values of $m$ are reached so the collisional dissipation becomes important and, indeed, dominant; this $m$-cutoff is clearly visible in the rightmost plot of the second row of Fig. 4.

Let us estimate the velocity-space dissipation scale. Linearising Eq. (4) for a given $k_{y}$ we find that the electron free energy spectrum $E_{m}=\left|g_{m}\right|^{2} / 2$ satisfies [8]

$$
\frac{\partial E_{m}}{\partial t}=-\left|k_{y}\right| \frac{B_{y}}{B_{z}} v_{\text {the }} \frac{\partial}{\partial m} \sqrt{2 m} E_{m}-2 \nu_{\text {coll }} m^{4} E_{m} .
$$

Setting $\partial E_{m} / \partial t=2 \gamma E_{m}$, we obtain

$$
E_{m}=\frac{C\left(k_{y}\right)}{\sqrt{m}} \exp \left[-\left(\frac{m}{m_{\gamma}}\right)^{1 / 2}-\left(\frac{m}{m_{c}}\right)^{9 / 2}\right],
$$

where $m_{\gamma}=\left[k_{y} B_{y} / B_{z} v_{\text {the }} /(2 \sqrt{2} \gamma)\right]^{2}, \quad m_{c}=$ $\left[9 /(2 \sqrt{2}) k_{y} B_{y} / B_{z} v_{\text {the }} / \nu_{\text {coll }}\right]^{2 / 9}$ and $C\left(k_{y}\right)$ is some $k_{y}$-dependent constant. As is evidenced by Fig. 1(a), $\gamma$ is independent of collisions and thus so is $m_{\gamma}$. Therefore, while the mode is strongly growing, $m_{\gamma}<m_{c}$ and so the collisional cuttoff cannot be reached. This explains why the peak of the dissipation rate must occur later than that of the reconnection rate. As reconnection proceeds into the saturation regime, $\gamma \rightarrow 0$, so, regardless of how small $\nu_{\text {coll }}$ is, eventually $m_{\gamma}>m_{c}$ and, from then onwards, the Hermite spectrum cutoff is determined by $m_{c}$. In our simulations, this happens at $t \approx 26 \tau_{A}$, with no appreciable dependence on collisionality $(M)$,

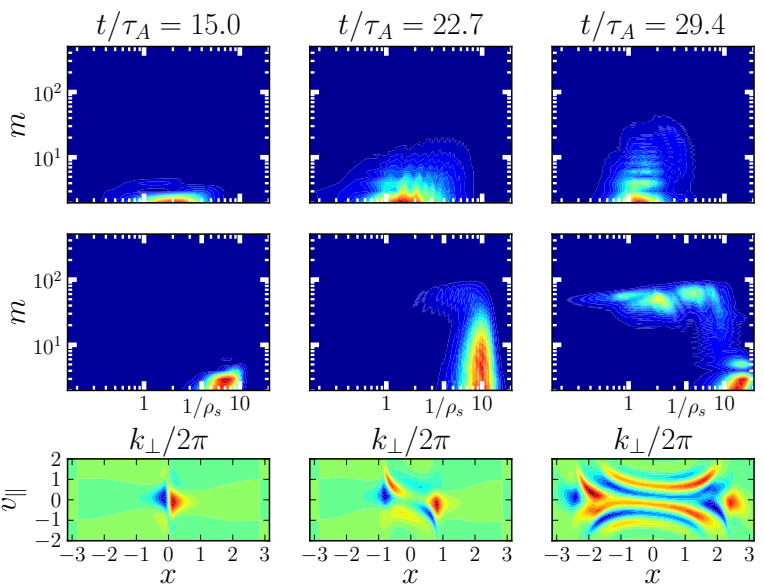

FIG. 4. Electron free energy spectrum (top row), dissipation spectrum (middle row) and a cut at $y / a=2.3$ (cf. Fig. 2) of the distribution function (bottom row) at the early nonlinear stage (left) and at the peaks of the reconnection rate (center) and dissipation rate (right) for a run with $\Delta^{\prime} a=20$ and $M=500$.

because the decrease of $\gamma$ is fast [see Fig. 1(a)]. The inset in Fig. 5 shows the time lag between the peaks of the reconnection and dissipation rates vs. M. The logarithmic dependence is due to the fast decay of $\gamma$, and the consequent rapid increase of $m_{\gamma} \sim \gamma^{-2}$ to overtake $m_{c}$, thus enabling dissipation. The weak dependence of the lag on collisions implies that dissipation occurs in finite time even for weak collisionality.

The value of $m=m_{\text {peak }}$ at which most energy is dissipated is the solution of $d\left(\nu_{\text {coll }} m^{4} E_{m}\right) / d m=0$, with $E_{m}$ given by Eq. (6) in the regime $m_{\gamma} \gg m_{c}$. This yields $m_{\text {peak }}=(9 / 7)^{2 / 9} m_{c}$. This expression, evaluated for $k_{y}=1$ [27], is compared in Fig. 5 with the numerically determined value of $m$ at which the dissipation peaks. The remarkable agreement that is obtained shows that the electron heating we observe is the result of linear phase mixing.

The phase mixing process discussed above is illustrated by the plots in the bottom row of Fig. 4 showing the electron distribution function $g_{e}$, taken at $y / a=2.3$ (i.e., inside the island; see Fig. 2). The progressive creation of finer scales in velocity-space, a textbook signature of phase mixing, is manifest (cf. [28, 29]).

Conclusions. This Letter presents the first detailed investigations of electron heating caused by magnetic reconnection in strongly magnetized, weakly collisional plasmas. Using a novel fluid-kinetic framework [8], we were able to show that linear phase mixing/Landau damping is the main mechanism for energy conversion and electron heating. Reconnection and electron heating are causally related, but temporally and spatially disconnected: heating happens after most flux has reconnected, and along the island separatrices, not in the current sheet. Our other key conclusions are: (i) 


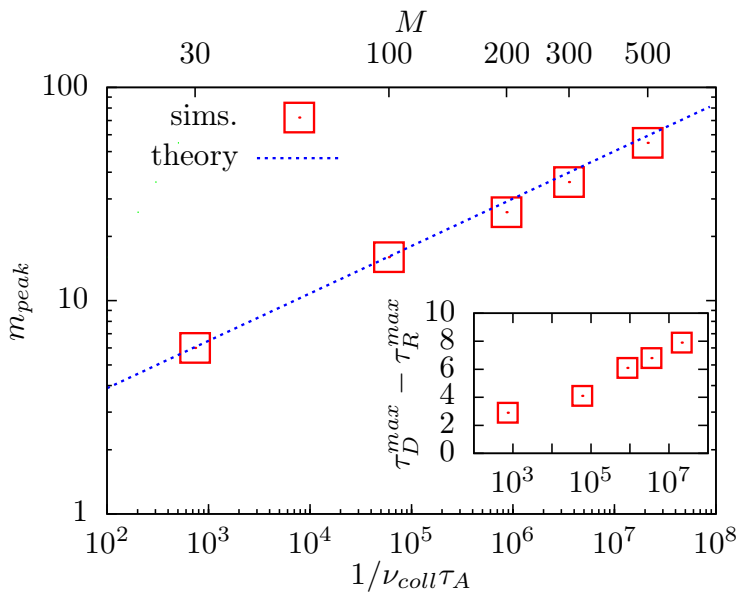

FIG. 5. Value of $m$ at which most energy is dissipated, $m_{\text {peak }}$, as a function of collisionality, $\nu_{\text {coll }}$. Inset: time lag between the peak of the reconnection rate $\left(\tau_{R}^{\max }\right)$ and the peak of the dissipation rate, $\left(\tau_{D}^{\max }\right)$, as a function of $\nu_{\text {coll }}$.

the maximum reconnection rate in the asymptoticallylarge-guide-field limit is as fast as in the no-guide-field limit, $c E_{\max } \sim 0.2 v_{A} B_{y, 0}$, provided that the system is large enough; (ii) the saturation amplitude in the kinetic (weakly collisional) regime is identical to that in MHD (collisional) systems [6], as long as the island is large compared to the kinetic scales. The electron inertia scale appears to provide the lower boundary on the saturation amplitude - this result may be important to the understanding of magnetized turbulence (e.g., [30-33]), as it effectively sets the minimum fluctuation amplitude.

Acknowledgments. This work was supported by Fundação para a Ciência e Tecnologia (Ciência 2008 and Grant no. PTDC/FIS/118187/2010), by the European Communities under the contracts of Association between EURATOM and IST and EURATOM and CCFE, and by the Leverhulme Trust Network for Magnetised Plasma Turbulence. The views and opinions expressed herein do not necessarily reflect those of the European Commission. Simulations were carried out at HPC-FF (Juelich), Jugene (PRACE) and Ranger (NCSA).

[1] E. G. Zweibel and M. Yamada, Ann. Rev. Astron. Astrophys. 47, 291 (2009).

[2] H. P. Furth, J. Killeen, and M. N. Rosenbluth, Phys. Fluids 6, 459 (1963).

[3] P. H. Rutherford, Phys. Fluids 16, 1903 (1973).

[4] D. F. Escande and M. Ottaviani, Phys. Lett. A 323, 278 (2004).

[5] F. Militello and F. Porcelli, Phys. Plasmas 11, L13 (2004).

[6] N. F. Loureiro, S. C. Cowley, W. D. Dorland, M. G. Haines, and A. A. Schekochihin, Phys. Rev. Lett. 95, 235003 (2005).
[7] N. F. Loureiro, R. Samtaney, A. A. Schekochihin, and D. A. Uzdensky, Phys. Plasmas 19, 042303 (2012).

[8] A. Zocco and A. A. Schekochihin, Phys. Plasmas 18, 102309 (2011).

[9] E. A. Frieman and L. Chen, Phys. Fluids 25, 502 (1982).

[10] J. A. Krommes, Phys. Rep. 360, 1 (2002).

[11] G. G. Howes, S. C. Cowley, W. Dorland, G. W. Hammett, E. Quataert, and A. A. Schekochihin, Astrophys. J. 651, 590 (2006).

[12] I. G. Abel, G. G. Plunk, E. Wang, M. Barnes, S. C. Cowley, W. Dorland, and A. A. Schekochihin, ArXiv e-prints (2012), arXiv:1209.4782 [physics.plasm-ph].

[13] We keep the collision operator in Eq. (3), but ignore the resistive term $\eta \nabla_{\perp}^{2} A_{\|}$on the RHS of Eq. (2). This is formally inconsistent, since $\eta=\nu_{e i} d_{e}^{2}$; in practice, the only effect of setting $\eta=0$ is allowing the use of larger values of $\nu_{e i}$ than would otherwise be required in order to access the collisionless regime.

[14] T. J. Schep, F. Pegoraro, and B. N. Kuvshinov, Phys. Plasmas 1, 2843 (1994).

[15] N. F. Loureiro and G. W. Hammett, J. Comp. Phys. 227, 4518 (2008).

[16] M. Ottaviani and F. Porcelli, Phys. Rev. Lett. 71, 3802 (1993).

[17] B. Rogers and L. Zakharov, Phys. Plasmas 3, 2411 (1996).

[18] G. Valori, D. Grasso, and H. J. de Blank, Phys. Plasmas 7, 178 (2000).

[19] J. Birn et al., J. Geophys. Res. 106, 3715 (2001).

[20] B. N. Rogers, R. E. Denton, J. F. Drake, and M. A. Shay, Phys. Rev. Lett. 87, 195004 (2001).

[21] B. N. Rogers, S. Kobayashi, P. Ricci, W. Dorland, J. Drake, and T. Tatsuno, Phys. Plasmas 14, 092110 (2007).

[22] D. A. Uzdensky and R. M. Kulsrud, Phys. Plasmas 13, 062305 (2006).

[23] Note that in none of our runs did we observe the current sheet becoming unstable to plasmoid formation, though this may be because even our largest system is in fact not large enough: the longest box is $L_{y} / a=3.785 \pi$ (for $\left.\Delta^{\prime} a=54\right)$; this run yields the maximum ratio of the current sheet length to $\rho_{s}$ of $\sim 8$, too small to expect a transition to multiple X-line reconnection [34, 35].

[24] R. D. Sydora, Phys. Plasmas 8, 1929 (2001).

[25] If we use a (formally incorrect) isothermal closure, most of the dissipation occurs via Ohmic heating.

[26] This is why the reconnection rate should be independent of the details of the dissipation: indeed, the same rate should be obtained even in dissipation-free Hamiltonian models [28, 36-39], although they would not obtain the same energy partition that we report here.

[27] We chose $k_{y}=1$ because this is the characteristic scale of the energy transfer, as is clear from the top row of Fig. 4. Note, however, that $m_{c} \sim k_{y}^{2 / 9}$, a fairly weak dependence.

[28] D. Grasso, F. Califano, F. Pegoraro, and F. Porcelli, Phys. Rev. Lett. 86, 5051 (2001).

[29] T. V. Liseikina, F. Pegoraro, and E. Y. Echkina, Phys. Plasmas 11, 3535 (2004).

[30] H. Doerk, F. Jenko, M. J. Pueschel, and D. R. Hatch, Phys. Rev. Lett. 106, 155003 (2011).

[31] W. Guttenfelder et al., Phys. Rev. Lett. 106, 155004 (2011). 
[32] W. M. Nevins, E. Wang, and J. Candy, Phys. Rev. Lett. 106, 065003 (2011).

[33] D. R. Hatch, M. J. Pueschel, F. Jenko, W. M. Nevins, P. W. Terry, and H. Doerk, Phys. Rev. Lett. 108, 235002 (2012).

[34] H. Ji and W. Daughton, Phys. Plasmas 18, 111207 (2011).

[35] Y.-M. Huang, A. Bhattacharjee, and B. P. Sullivan, Phys. Plasmas 18, 072109 (2011).

[36] B. N. Kuvshinov, F. Pegoraro, and T. J. Schep, Phys. Lett. A 191, 296 (1994).
[37] E. Tassi, P. J. Morrison, F. L. Waelbroeck, and D. Grasso, Plasma Phys. Control. Fusion 50, 085014 (2008).

[38] F. L. Waelbroeck, R. D. Hazeltine, and P. J. Morrison, Phys. Plasmas 16, 032109 (2009).

[39] D. Del Sarto, C. Marchetto, F. Pegoraro, and F. Califano, Plasma Phys. Control. Fusion 53, 035008 (2011). 